\title{
The roots of complex problems
}

\section{Fearless Symmetry: Exposing the Hidden Patterns of Numbers \\ by Avner Ash \& Robert Gross \\ Princeton University Press: 2006. 302 pp. \\ $\$ 24.95, £ 15.95$}

\section{Timothy Gowers}

If you ask somebody who knows a little mathematics what is meant by the symbol $i$, they will probably tell you, correctly, that it stands for the square root of -1 . But there is a subtlety to the question that is easy to overlook. Suppose that you pedantically point out that there are two square roots of -1 . The response is likely to be that the other square root is $-i$. But now comes a much harder question: which square root is $i$ and which one is $-i$ ?

The more one thinks about this question, the more one realizes that it does not have an answer. In fact, the question doesn't really make sense, and mathematicians even have a way of proving that it doesn't make sense. The rough idea of the proof is this. If $z$ is any complex number, written in its usual form $a+i b$, where $a$ and $b$ are real numbers, then we define the complex conjugate of $z$ to be $a-i b$, and denote this number by $\bar{z}$. It can then be proved that, for any two complex numbers $z$ and $w, \overline{z+w}=\bar{z}+\bar{w}$ and $\overline{z w}=\bar{z} \bar{w}$. In mathematical terminology, the function that takes each complex number to its conjugate is an automorphism, because it 'preserves' the basic arithmetical operations.

Because of this automorphism, there is no true mathematical sentence about $i$ that is not equally true when all occurrences of $i$ (both implicit and explicit) are replaced by $-i$. This is the sense in which $i$ and $-i$ are indistinguishable and is the reason that one cannot answer the question: "Which square root of -1 is $i$ ?" By contrast, it is possible to distinguish between 1 and -1, for example, as the

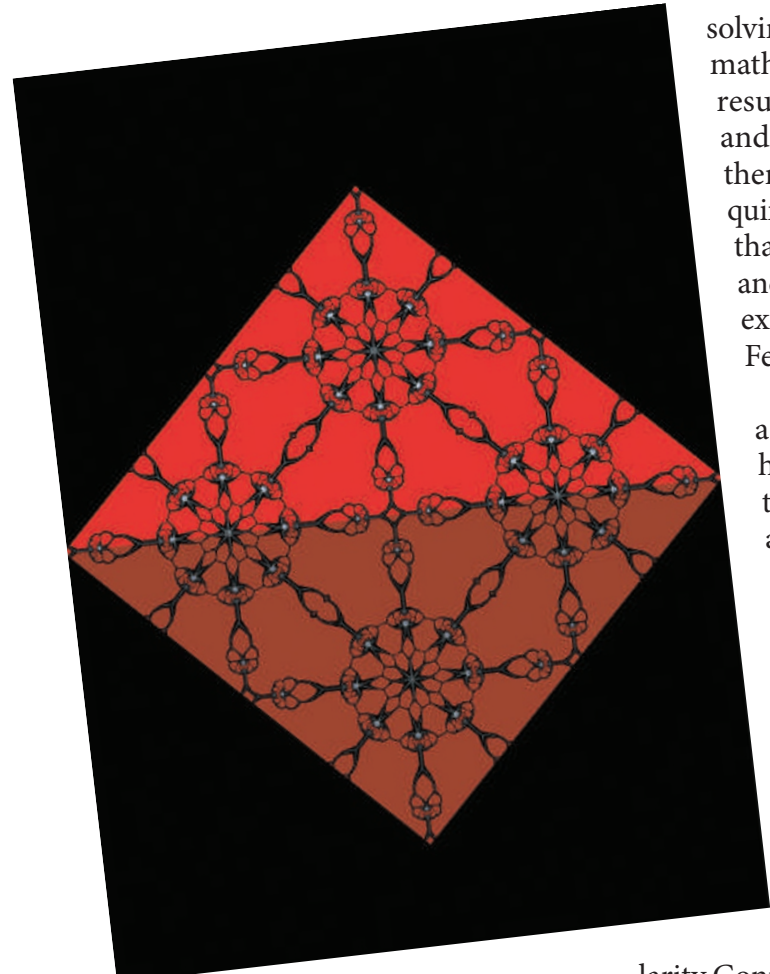

solving some of the deepest problems in mathematics. A notable example is the result, obtained by Niels Henrik Abel and extended by Évariste Galois, that there is no formula for solving general quintic equations, or at least no formula that involves just algebraic operations and taking roots. A much more recent example is Andrew Wiles' solution of Fermat's Last Theorem.

Fearless Symmetry started life as an expository paper intended to help mathematicians in other areas to understand Wiles' remarkable achievement. It then grew into a book, and to make it more accessible the authors added a lot of background material. The result is that to begin with there are plenty of gentle sentences such as: "We start our consideration of groups by thinking about a beautiful perfect sphere, one foot in radius, made of pure marble." By the end these are accompanied by sentences such as: "By the Modu-

Polynomiographs such as this one, Acrobats, are created using mathematical formulae.

square of 1 is itself, but the square of -1 is not itself.

Automorphisms such as this are the 'fearless symmetries' of the title of Avner Ash and Robert Gross's book. They can be thought of as symmetries because they are transformations of a mathematical object (which happens to be algebraic rather than geometrical) that leave its important properties unchanged. It turns out that understanding these symmetries in more complicated situations is the key to

\section{NEW IN PAPERBACK}

\section{The Evolution-Creation Struggle}

by Michael Ruse (Harvard University Press, $£ 10.95$, $€ 14.40, \$ 16.95$ )

"In this book, Ruse aims not to attack but to understand. For that he wisely turns to history - specifically to the history of evolutionary theory." John Hedley Brooke, Nature 437, 815-816 (2005).

\section{The Revenge of Gaia}

by James Lovelock (Penguin, E8.99)

"James Lovelock... offers his take on the future of energy.
In brief, a vigorous turn towards nuclear power will be necessary to prevent the catastrophic climatic changes caused by an increase in atmospheric carbon dioxide." Tyler Volk, Nature 440, 869-870 (2006).

\section{Thinking With Animals:} New Perspectives on Anthropomorphism edited by Lorraine Daston \& Gregg Mitman (Columbia University Press, \$25, £16)

"An unusual book that will surely join the growing literature on consciousness, animal cognition and the continuity between human and animal minds." Juliette Clutton-Brock, Nature 434, 958-959 (2005)

\section{Terrors of the Table: The} Curious History of Nutrition by Walter Gratzer (Oxford University Press, $£ 9.99$, \$16.95)

"[Gratzer's] purpose is 'to astonish, to instruct and, most especially, to entertain'. And what could possibly be more entertaining than the history of nutrition?" Marion Nestle, Nature 438, 425-426 (2005). larity Conjecture, there is a cuspidal normalized newform $f$ of level $N$ and weight 2 such that for all primes $w$ that are not factors of $N$, $a w(f)=a w(E)$, and hence these pairs of integers are congruent modulo $p$." In between, the level of sophistication rises steadily. A typical reader, therefore, will find that the book starts by covering familiar ground, then becomes interesting and informative, and finally becomes too difficult to understand. Where these transitions take place will vary from reader to reader: I learned a lot from about the middle third of the book and not much from the outer thirds. But that was enough to make it worth reading, and perhaps one day I will be ready to have another go at the later chapters.

One small disappointment was a section promisingly entitled: "Digression: What is so great about elliptic curves?" Anybody who has followed the story of the proof of Fermat's Last Theorem will have heard that elliptic curves are very important, but it is not at all obvious from the definition here why they should be. Eager for a better understanding, I turned to that section only to find that the answer is that elliptic curves are incredibly useful to number theorists. There are less question-begging answers later in the book, but by then the going is rather tough.

But that was just a digression. In general, the authors are to be admired for taking a very difficult topic and making it, if not fully accessible, then certainly more accessible than it was before.

Timothy Gowers is at the Centre for Mathematical Sciences, University of Cambridge, Wilberforce Road, Cambridge CB3 OWB, UK. 